\title{
Integration of the "smart home" concept into the infrastructure of the modern city
}

\author{
$I V$ Eliseev $^{1}, B M$ Shifrin, $M A$ Novikova and $V A$ Sokolova
}

Saint-Petersburg State Forestry University

\begin{abstract}
Currently, the experience of implementing building automation systems shows that many leading manufacturers of equipment and software in this area are trying to develop their own protocols, standards and interfaces for data transfer and control at the lower level of interaction, which generally complicates and increases the cost of implementing projects using equipment and technologies of various manufacturers, and also lowers the level of security and fault tolerance of such systems. rThe main idea is to integrate various elements of a distributed system for managing the infrastructure of objects into a single network data transfer infrastructure using standards that describe the interaction in wired and wireless local area networks.
\end{abstract}

\section{Introduction}

In modern intelligent buildings automation and control systems occupy a key place at the socio-cultural, sports facilities, and transport. They ensure the interconnection of all engineering equipment and systems responsible for the safety, comfort and access of a large number of users to information resources and augmented reality technologies.

To ensure a given level of comfort, safety, reliability, information support, the automated technical systems must perform the following basic functions:

- To manage the operation of functional elements;

- To turn on and off electric motor drives;

- To monitor the equipment;

- To generate alarms: ventilation control, heating control, lighting control, etc.;

- To ensure security at the perimeter of the facility;

- To analyze the video stream from the security cameras.

A number of studies in recent years have shown a steady trend towards an increase in the share of the cost and volume of engineering systems and automation systems in the total cost of construction projects. The development of this trend to the present moment has led to a qualitative change in the place and role of automation and building management systems on one hand, and the concept of mutual linking of engineering equipment of facilities and organizational and technical solutions for operation using automation and control systems of buildings and other objects on the other hand.

We single out several basic functions of a typical management system of an intelligent building:

- The ability to control all building systems using keypads;

- The ability to control using smart switches and touch graphic consoles;
- Control of all lighting of the facility;

- Scenario control: customizable indoor lighting scenes, automatic lighting control by time and events;

- Lighting control of corridors and stairs by motion sensors.

- Management of motorized curtains;

- Zonal climate control;

- Control of hoods and the general ventilation system of the facility;

- Creation of a unified multimedia system with the ability to switch signals for organizing mass access to resources;

- Power load management;

- Security system management;

- Differentiation of access rights to resources and management functions;

- Organization of a centralized or distributed data storage system;

- Providing video monitoring at the facility with the possibility of automated intelligent data analysis from cameras in real time and event management [1-3].

Most of the functionality of the Smart Home systems is in demand and can be implemented in the management of smart city infrastructure. Besides this, building automation and control systems form the basis for creating new services for users. This is expressed in increasing the consumer attractiveness of intelligent buildings and the urban environment, which is manifested, in particular, in reducing insurance risks by increasing resistance to various destabilizing factors and lowering operating costs, i.e. in increasing the efficiency of intelligent objects compared to traditional solutions. In this regard, the main idea is to integrate various elements of a distributed system for managing the infrastructure of objects into a single network data transfer infrastructure using standards that describe the interaction in wired and wireless local area networks.

\footnotetext{
*Corresponding author: yeliseyef@yandex.ru
} 
To implement this idea, an analysis of the existing solutions for arrangement of distributed building automation systems is performed. It was revealed that one of the drawbacks of various Smart Home systems is the lack of connectivity of sensors, actuators, information display, monitoring and control devices with the system core.

Currently, the experience of implementing building automation systems shows that many leading manufacturers of equipment and software in this area are trying to develop their own protocols, standards and interfaces for data transfer and control at the lower level of interaction, which generally complicates and increases the cost of implementing projects using equipment and technologies of various manufacturers, and also lowers the level of security and fault tolerance of such systems.

We have analyzed all the basic requirements that a data transmission system must meet. The main ones are:

- Minimum amount of construction and installation work should be carried out during its implementation;

- It should be created on the basis of inexpensive components;

- It should provide reliable identification of active devices;

- It should ensure a high level of data transmission quality, be resistant to interference;

- It should have sufficient bandwidth;

- It should be able to maintain operability in case of damage to part of the system and/or head units;

- It should provide the widest possible range of devices, as well as support the easy integration of new devices into the system.

Currently, there are several popular options for organizing networking at the physical level:

- Wireless;

- Through specially laid wires (usually twisted pair);

- Using power cables;

- GSM standards [4].

Each of the listed options has certain advantages and disadvantages, therefore, to ensure the universality and flexibility of device interaction in the infrastructure management systems of urban facilities, it is proposed to use the standards and protocols of local Ethernet computing networks in combination with a wired and wireless data transmission medium [5].

This approach will ensure:

- Universality in terms of control devices choice;

- Savings on the installation and laying of communication lines, since most often SCS is already incorporated into the project or exists;

- The possibility of using both wired and wireless data transfer technologies without the need to develop interaction schemes;

- Ensuring the fault tolerance of a data transmission system based on standard protocols of the second and third levels of an open network model;

- Ensuring a high level of security, multi-level access control and intrusion protection.

The accumulated experience in designing and cooperation with leading manufacturers of equipment for organizing local networks make it possible to implement automation systems for objects of any complexity, taking into account all the requirements and the most varied wishes of project customers.

Another key problem is that the user is forced to adapt to the logic of the software and hardware complex, and not vice versa. This, in particular, is applied to interfaces. In the Smart Home system, the most natural interface can be achieved by voice commands, which allows one to identify the user by his voice. Thus, the modern Smart Home system should include a fullfledged voice interface, which, together with artificial intelligence, should form some kind of pseudo-identity with which the user will associate his Smart Home. All these functions should form the intellectual core, which, in fact, is a key element of the system [6].

\section{Materials and methods}

As part of this work, topologies of distributed data transmission networks were developed and tested for various objects, namely: university campus, schools of general education, kindergartens, business centers, museums, outdoor public areas, hotels, private houses and cottage villages, office buildings.

The practical significance is in the development and use of universal approaches to building the network infrastructure of various objects in order to simplify the design process and reduce costs both for design and implementation of building automation systems, As the final result, this allows one to implement a wide range of functions and capabilities within a single network infrastructure.

\section{Results}

In the framework of this work, the following scientific and technical results were obtained:

1. Network solutions based on ZYXEL equipment for several commercial and private facilities (buildings) have been developed to ensure comfort, safety of people and increase the resistance of intelligent buildings to external factors. The projects were carried out jointly by several system integrators working in the North-west Russian market in the field of development and implementation of building automation systems,

2. A scientific and educational center of network technologies for data transfer based on Saint-Petersburg State Forestry University. The main activities of the educational center are:

- Training and scientific work of students, graduate students and young scientists in the field of research and development of data transfer technologies and equipment for building LAN-WAN networks;

- Research in the field of distributed data storage models and organization of access to them;

- Development of virtual models of the functioning of complex multi-level data transmission networks.

As a result of analysis and study of various areas of development of wired and wireless data transfer technologies, issues related to the organization of corporate security and network resiliency, methods for ensuring quality of service and prioritization, the 
following basic sections of the educational program "network technologies" are formulated:

- Construction of enterprise networks based on L2 and L3 switches;

- Network security;

- Construction of wireless access networks;

- Design and maintenance features of networks of telecom operators;

- Features of networks construction for organizing perimeter security using IP video monitoring;

- Dynamic routing protocols in large networks;

- Protocols for organizing virtual private networks;

- Construction of high-density wireless networks [5].

\section{Discussion}

\subsection{Commercial prospects of this work}

The obtained scientific results will ensure:

- Universality in terms of choice of management systems devices for building infrastructure and other social facilities;

- Savings on installation and laying of communication lines, since most often SCS is already included in the project or exists;

- Reducing the time of design, installation and commissioning;

- Increasing the consumer attractiveness of intelligent buildings, expressed, in particular, in reducing insurance risks by increasing the resistance of intelligent buildings to various destabilizing factors and lowering operating costs, i.e. in increasing the efficiency of intelligent buildings compared to traditional solutions, ensuring a high level of security, multi-level access control and protection against intrusions [7-9].

Based on the emerging growth trend in the housing market, in the field of small and medium-sized businesses, as well as in the social and cultural fields, there has been a steady demand for the development and implementation of intelligent building management systems.

\subsection{Expected effect}

A universal approach to construction the network infrastructure of standard facilities can increase the economic attractiveness of projects in the field of building automation, creating an accessible environment for people with disabilities, ensuring safety at mass sports facilities, increasing comfort, safety, etc. In this regard, there is a need for the training of highly qualified personnel in the field of automation and control, data transmission networks, programming, circuitry and electronics.

The increase in demand for intelligent management systems for private, commercial, social and cultural facilities will increase the contribution of the IT industry to the growth of gross regional product.

\section{Conclusions}

Based on the required functions assigned to building automation systems and a wide range of customers' wishes, it is proposed to introduce typical topologies of distributed data networks. The aim of these actions is to ensure reliable interaction of all devices with the system core with the possibility of flexible scaling of the project within the selected network infrastructure and the implementation of wide functionality in the field of ensure the comfort and safety of people.

\section{References}

1.Principles of development of automatic control system and dispatching control of ventilation. Sokolova V.A., Shifrin B.M., Eliseev I.V., Chernykh L.G. In the collection: February readings Collection of materials of a scientific and practical conference based on the results of research work of 2017 by lecturers of the Syktyvkar Forest Institute. 2018. P. 35-37.

2. Implementation of the project of management and control of household appliances in a smart home. Aitbayeva K.Zh., Asainova A.Zh. Aktual'naya nayka. 2018. № 10 (15). P. 17-21.

3. Feoktistov D.Yu., Volnikov M.I. Automation in the modern house - "Smart House". In the collection: Innovative ideas of young researchers for the agroindustrial complex of Russia. Collection of articles of the International scientific-practical conference of young scientists. 2018. P. 267-269.

4. Shadrin D.V., Scherbakov M.S., Pochufarov A.O.Wireless data transmission protocol for the "Smart House" system with support of the modular network structure. In the book: New Information Technologies in the Study of Complex Structures Materials of the Twelfth Conference with International Participation. 2018. P. 48-49.

5.Platunova S.M., Eliseev I.V., Avksentieva E.Yu. Ethernet switches L2\&L3. Design, configuration, diagnostics of data transmission networks. Textbook on disciplines: Theory of design of computer systems, Computer networks and telecommunications, Architecture and hardware of computer networks. - St. Petersburg: NRU ITMO, 2018. 87 p.

6. Afanasyev G.I., Belkov A.D., Kovaleva N.A. Multimedia in the "Smart House". Territory of innovation. 2018. No 8 (24). P. 7-15.

7.Home-switches from https://www.zyxel.com/ru/ru/products services/homeswitches.shtml? $\mathrm{t}=\mathrm{c}$

8. Wi-Fi systems from ZYXEL. https://www.zyxel.com/ru/ru/products_services/service provider-wifi_system.shtml? $\mathrm{t}=\mathrm{c}$

9. Security Farewalls https://www.zyxel.com/ru/ru/products_services/smbsecurity_firewalls.shtml? $\mathrm{t}=\mathrm{c}$ 